\title{
STABILITY OF NEUTRAL DELAY DIFFERENTIAL EQUATIONS WITH APPLICATIONS IN A MODEL OF HUMAN BALANCING
}

\author{
Alexander Domoshnitsky*, Shai Levi, Ron Hay Kappel, \\ Elena Litsyn AND ROMAN YAVICH
}

\begin{abstract}
In this paper the exponential stability of linear neutral second order differential equations is studied. In contrast with many other works, coefficients and delays in our equations can be variable. The neutral term makes this object essentially more complicated for the study. A new method for the study of stability of neutral equation based on an idea of the Azbelev W-transform has been proposed. An application to stabilization in a model of human balancing has been described. New stability tests in explicit form are proposed.
\end{abstract}

Mathematics Subject Classification. 34K20, 34K40, 34K12, 34K21.

Received September 26, 2020. Accepted January 20, 2021.

\section{INTRODUCTION}

The main object of this paper is the second order neutral delay differential equation

$$
x^{\prime \prime}(t)+\sum_{i=1}^{m} q_{i}(t) x^{\prime \prime}\left(t-\phi_{i}(t)\right)+\sum_{i=1}^{m} a_{i}(t) x^{\prime}\left(t-\tau_{i}(t)\right)+\sum_{i=1}^{m} b_{i}(t) x\left(t-\theta_{i}(t)\right)=f(t), \quad t \in[0, \infty)
$$

with the corresponding initial functions

$$
x(\xi)=\varphi(\xi), x^{\prime}(\xi)=\psi(\xi), x^{\prime \prime}(\xi)=\eta(\xi) \text { for } \xi<0,
$$

defining what should be set into the equation instead of $x\left(t-\theta_{i}(t)\right), x^{\prime}\left(t-\tau_{i}(t)\right)$ and $x^{\prime \prime}\left(t-\phi_{i}(t)\right)$ in the case of $t-\theta_{i}(t)<0, t-\tau_{i}(t)<0$ or $t-\phi_{i}(t)<0$ respectively. For simplicity and without loss of generality in the study of the exponential stability we can consider the zero initial function [1]

$$
x(\xi)=x^{\prime}(\xi)=x^{\prime \prime}(\xi)=0 \text { for } \xi<0 .
$$

We assume that $f, a_{i}, b_{i}$ are measurable essentially bounded functions $[0, \infty) \rightarrow(-\infty, \infty)$. Concerning the delays $\theta_{i}(t), \tau_{i}(t), \phi_{i}(t)$ we assume that they are essentially bounded measurable non-negative functions. We also assume

Keywords and phrases: Delay equations, uniform exponential stability; Cauchy function; exponential estimates of solutions and Cauchy functions.

Department of Mathematics, Ariel University, Ariel, Israel.

* Corresponding author: adom@ariel.ac.il 
that the measure of the values such that $t-\phi_{i}(t) \in \Omega$, where $\Omega$ is a set of a zero lebesgue measure is of a zero measure. It is clear, for example, that $t-\phi_{i}(t)$ cannot be a constant for any interval from $[0, \infty), i=1, \ldots, m$. Note that there are many results for the case of $q_{i}(t) \equiv 0, i=1, \ldots, m, t \geq 0$ and only a fit results for the case of non-zero $q_{i}(t)$ in the study of stability.

Neutral functional differential equations were considered in the known books $[1,9,12,13]$ (see also the bibliographies therein), where existence and uniqueness of solutions and especially stability and oscillation results for these equations were obtained. Applications of neutral differential equations were presented in the book [16]. The human self-balancing models were studied in the papers [14, 22-24]. Various applications of equation (1.1) in the case of $q_{i}=0,1 \leq i \leq m$ can be found, for example, in the theory of self-excited oscillations, in oscillation processes in a vacuum tube, in dynamics of an autogenerator, in description of processes of infeed grinding and cutting (see the book [16]), in position control in mechanical engineering (for example, the model of container crane: it is important for the crane to move rapidly, the payload may oscillate and, as a result, the crane operator can loose control of the payload), in electromechanical systems, in combustion engines [10]. Equation (1.1) with $q_{i}=0$ is of interest in machine tool analysis, in biology in explaining self-balancing of the human body and in robotics in constructing biped robots [11] (see bibliography in [5]). The problem of stabilizing the rolling of a ship by the activated tanks method in which ballast water is pumped from one position to another was reduced in [18] to analysis of stability of the second order equation (1.1) in case of $q_{i}=0$. The control based on values of acceleration at previous moments of time, which forms the "neutral term" in (1.1) could look very naturally in these models. Results on stability of systems of neutral delay differential systems were obtained in $[1,2,21]$. Models with neutral delay differential equations in mechanical problems were constructed and studied in [23, 24]. Stability of equation (1.1) using positivity-based approach was studied in the recent paper [3].

Our development of the known results on stability of neutral equations can be seen in the following directions. Although the study of delay equations historically started with equations with constant delays $\theta_{i}, \tau_{i}$ $(i=1, \ldots, m)$, this case does not look naturally in modeling control of technical devices. The first development is in the study of equation with variable delays in the frame of applications. In real applications the measurements of the process at discrete moments of times are used. It means that the deviating arguments are actually of the form $t-\theta_{i}(t)=g_{i}, t-\tau_{i}(t)=h_{i}, t \in\left(t_{i}, t_{i+1}\right), i=1,2, \ldots, m$, where $g_{i}, h_{i}$ are constants $(i=1,2, \ldots, m)$. Simulation of control of smart prostheses, which is one of most important applications of the human balancing model, discussed below in Section 4, is based in fact on the values of angles and speeds of their changes is also based on delays of this sort. In many cases variable delays appear naturally in mathematical models. For example, control can depend on geometry of electromechanical device, for instance on the distance between sensors, mechanical and control blocks. If the distances can change we come to variable delays in the model.

The second direction of our development of the model of human balancing $[14,23]$ is in considering variable coefficients. The mechanical stiffness in biological systems is rather periodic than constant and this in a corresponding sense explains considering variable coefficients. But there is also another reason.

Below, discussing the model of human balancing in Section 4, we explain additional ways of appearing variable coefficients in this model. It will be demonstrate, for instance, that a combination of small external unpredictable influences and nonlinearities can imply variable coefficients in the model. A "small" external force can change the position of the stationary point, and this could lead to the fact that the coefficients of the system linearized in the neighborhood of this new stationary point change because they depend on the solution. Example 4.1 demonstrates that a system without a right-hand side linearized in the neighborhood of the solution $x=0$ can be exponentially stable, and a system linearized in the neighborhood of $x=\delta$, where $\delta$ is a "small" constant, may be Lyapunov unstable. It is natural in these cases to consider equations with variable coefficients instead of equations with constant ones.

If a person falls, then the theoretical fact about stability gives nothing. This demonstrated the importance of considering non-homogeneous equations. In practical tasks, the influence of the right-hand side on the solution should be evaluated. It is necessary to obtain a dependence of the solution on the right-hand side bounded by corresponding constants. We will get such estimates in Section 3. This is the third direction in the development of human balancing model. 
The principal development of the results presented in [3] is in considering cases of oscillation behavior of solutions to neutral equation (1.1), where positivity-based approach does not work (see Rem. 3.3). The results of [3] cannot be used for the model of human balancing, see, for example, equation (4.10), in which the coefficient of $\varphi(t)$ is negative and the assumption of the main result of [3] about exponential stability of the ordinary part $\varphi^{\prime \prime}+A \varphi^{\prime}+B \varphi=0$ is not fulfilled. Even in the case of $\theta_{2}=0$ in equation (4.10), the main result of [3] does not work in the cases of such small $q_{i}$ and large coefficients of $x$ and $x^{\prime}$.

In this paper we propose a method for stability analysis of neutral equations. This method leads us to conditions on smallness of delays that is naturally from the physical point of view. Our paper is built as follows. In Section 2 we describe known results which are used in the proofs. In Section 3 we formulate results on stability of equation (1.1). In Section 4 we describe the known model of human balancing and our developments concerning variable delays and coefficients from mechanical point of view. In Section 5 applications of our results to estimates of delays in the model of human balancing are obtained. In Section 6 examples for estimate of acceleration coefficient for the human balancing are presented. In Section 7 we prove main results. In Section 8 discussion and open questions are presented.

\section{PRELiminary}

We understand a solution of equation (1.1) and (1.2) as a function $x:[0, \infty) \rightarrow(-\infty, \infty)$ with absolutely continuous on every finite interval derivative $x^{\prime}$ and essentially bounded second derivative $x^{\prime \prime}$ which satisfies this equation almost everywhere.

The general solution of equation (1.1) and (1.2) can be represented in the form [1]

$$
x(t)=\int_{0}^{t} C(t, s) f(s) \mathrm{d} s+x_{1}(t) x(0)+x_{2}(t) x^{\prime}(0),
$$

where $x_{1}(t), x_{2}(t)$ are two solutions of homogeneous equation (2.2), (1.2), where

$$
x^{\prime \prime}(t)+\sum_{i=1}^{m} q_{i}(t) x^{\prime \prime}\left(t-\phi_{i}(t)\right)+\sum_{i=1}^{m} a_{i}(t) x^{\prime}\left(t-\tau_{i}(t)\right)+\sum_{i=1}^{m} b_{i}(t) x\left(t-\theta_{i}(t)\right)=0, \quad t \in[0,+\infty)
$$

satisfying the conditions

$$
x_{1}(0)=1, x_{1}^{\prime}(0)=0, x_{2}(0)=0, x_{2}^{\prime}(0)=1,
$$

respectively.

The kernel $C(t, s)$ in representation (2.1) is called the Cauchy function of equation (1.1).

Note that that in the classical books on delay differential equations $[9,19]$, the homogeneous equations are considered as equation (2.2) with absolutely continuous initial function

$$
x(\xi)=\varphi(\xi), x^{\prime}(\xi)=\varphi^{\prime}(\xi), x^{\prime \prime}(\xi)=\varphi^{\prime \prime}(\xi), \text { for } \xi \leq t_{0},
$$

Let us formulate several definitions concerning stability.

Definition 2.1. [1] Equation (2.2) is uniformly exponentially stable if there exist $N>0$ and $\alpha>0$, such that the solution of (2.2), (2.4), where

$$
x(\xi)=\varphi(\xi), x^{\prime}(\xi)=\varphi^{\prime}(\xi), x^{\prime \prime}(\xi)=\varphi^{\prime \prime}(\xi), \xi<t_{0}, x\left(t_{0}\right)=x_{0}, x^{\prime}\left(t_{0}\right)=x_{0}^{\prime},
$$


satisfies the estimate

$$
\max \left\{|x(t)|,\left|x^{\prime}(t)\right|\right\} \leq N e^{-\alpha\left(t-t_{0}\right)} \max \operatorname{esssup}_{\xi \in\left(-\infty, t_{0}\right]}\left(|\varphi(\xi)|,\left|\varphi^{\prime}(\xi)\right|,\left|\varphi^{\prime \prime}(\xi)\right|\right), \quad t_{0} \leq t<\infty .
$$

where $N$ and $\alpha$ do not depend on $t_{0}$.

Definition 2.2. [1] The Cauchy function $C(t, s)$ of equation (1.1) satisfies the exponential estimate if there exist positive $N$ and $\alpha$ such that

$$
|C(t, s)| \leq N e^{-\alpha(t-s)},\left|C_{t}^{\prime}(t, s)\right| \leq N e^{-\alpha(t-s)}, \quad 0 \leq s \leq t<\infty .
$$

It is known that for equation (1.1) under our assumptions these two definitions are equivalent [1]: Equation $(2.2),(2.4)$ is uniformly exponentially stable if and only if the Cauchy function $C(t, s)$ satisfies the exponential estimate (2.6).

Our technique in the study of the exponential stability is based on the Bohl-Perron theorem: for equation (1.1) with bounded delays, the exponential estimate of the Cauchy function is equivalent to the fact that for every bounded right hand side $f$, the solution $x$ and its derivative $x^{\prime}$ are bounded [1].

\section{Formulations of main Results}

Let us consider the following ordinary differential equation

$$
x^{\prime \prime}(t)+A x^{\prime}(t)+B x(t)=z(t), \quad t \in[0, \infty), A>0, B>0,
$$

with constant positive coefficients $A$ and $B$, where $z$ is an essentially bounded measurable function.

Denote by $W(t, s)$ the Cauchy function of equation (3.1). It is known that for every fixed $s$ the function $W(t, s)$, as a function of the variable $t$, satisfies the homogeneous equation

$$
x^{\prime \prime}(t)+A x^{\prime}(t)+B x(t)=0, \quad t \in[s, \infty),
$$

and the initial conditions

$$
x(s)=0, x^{\prime}(s)=1 .
$$

We have to construct $W(t, s)$ in every one of the cases:

if $A^{2}>4 B$ then

$$
W(t, s)=\frac{1}{\sqrt{A^{2}-4 B}}\left(\exp \left[\frac{-A+\sqrt{A^{2}-4 B}}{2}(t-s)\right]-\exp \left[\frac{-A-\sqrt{A^{2}-4 B}}{2}(t-s)\right]\right)
$$

if $A^{2}=4 B$ then

$$
W(t, s)=(t-s) \exp \left[-\frac{A}{2}(t-s)\right]
$$

and if $A^{2}<4 B$ then

$$
W(t, s)=\frac{2}{\sqrt{4 B-A^{2}}} \exp \left[-\frac{A}{2}(t-s)\right] \sin \left(\frac{\sqrt{4 B-A^{2}}}{2}(t-s)\right) .
$$


It is known that the solution of the equation (3.1) which satisfies the initial conditions

$$
x(0)=0, x^{\prime}(0)=0,
$$

can be written in the form

$$
x(t)=\int_{0}^{t} W(t, s) z(s) \mathrm{d} s .
$$

Its derivatives are the following

$$
x^{\prime}(t)=\int_{0}^{t} W_{t}^{\prime}(t, s) z(s) \mathrm{d} s, \quad x^{\prime \prime}(t)=\int_{0}^{t} W_{t t}^{\prime \prime}(t, s) z(s) \mathrm{d} s+z(t) .
$$

Let us denote

$$
\begin{gathered}
\|W\|=\sup _{t \geq 0} \int_{0}^{t}|W(t, s)| \mathrm{d} s \\
\left\|W_{t}^{\prime}\right\|=\sup _{t \geq 0} \int_{0}^{t}\left|W_{t}^{\prime}(t, s)\right| \mathrm{d} s,\left\|W_{t t}^{\prime \prime}\right\|=\sup _{t \geq 0} \int_{0}^{t}\left|W_{t t}^{\prime \prime}(t, s)\right| \mathrm{d} s .
\end{gathered}
$$

Denote by $A_{i}$ and $B_{i}$ the average values of the coefficients $a_{i}(t)$ and $b_{i}(t)$ in equation (1.1) which can be obtained as $A_{i}=\lim _{t \rightarrow \infty} \frac{1}{t} \int_{0}^{t} a_{i}(s) \mathrm{d} s$ and $B_{i}=\lim _{t \rightarrow \infty} \frac{1}{t} \int_{0}^{t} b_{i}(s) \mathrm{d} s$. To connect equations (1.1) and (3.1) we suppose below that the coefficients $A$ and $B$ in equation (3.1) are the sums

$$
A=\sum_{i=1}^{m} A_{i}, \quad B=\sum_{i=1}^{m} B_{i}
$$

Denote also $\Delta a_{i}(t)=A_{i}-a_{i}(t),\left|\Delta a_{i}\right|^{*}=\operatorname{esssup}_{t \geq 0}\left|\Delta a_{i}(t)\right|, \Delta b_{i}(t)=B_{i}-b_{i}(t),\left|\Delta b_{i}\right|^{*}=\operatorname{esssup}_{t \geq 0}\left|\Delta b_{i}(t)\right|$, $\theta_{i}^{*}=\operatorname{esssup}_{t \geq 0} \theta_{i}(t), \theta^{*}=\max _{i=1, \ldots, m} \theta_{i}^{*}, \tau_{i}^{*}=\operatorname{esssup}_{t \geq 0} \tau_{i}(t), \tau^{*}=\max _{i=1, \ldots, m} \tau_{i}^{*},|q|=\operatorname{esssup}_{t \in[0, \infty)} \sum_{i=1}^{m}\left|q_{i}(t)\right|$.

Denote by $x(t)$ the solution of homogeneous equation (2.2) and by $X(t)$ the solution of non-homogeneous equation (1.1) with the same initial conditions, and $f^{*}=\operatorname{essup}_{t \geq 0}|f(t)|$.

Theorem 3.1. Let $A>0, B>0$ and the following inequality be fullfilled:

$$
\begin{aligned}
Q \equiv & \sum_{i=1}^{m}\left|A_{i}\right| \tau_{i}^{*}\left\{\| W_{t t}^{\prime \prime}||+1\right\}+\sum_{i=1}^{m}\left|\Delta a_{i}\right|^{*}|| W_{t}^{\prime}||+\sum_{i=1}^{m}\left|B_{i}\right| \theta_{i}^{*}|| W_{t}^{\prime}\left\|+\sum_{i=1}^{m}\left|\Delta b_{i}\right|^{*}|| W\right\| \\
& +|q|\left\{\| W_{t t}^{\prime \prime}||+1\right\}<1 .
\end{aligned}
$$

Then equation (2.2) is uniformly exponentially stable, and $|x(t)-X(t)|<\frac{\|W\|}{1-Q} f^{*}$.

Remark 3.2. Denoting $P=\|W\|, Q=\left\|W_{t}^{\prime}\right\|, R=\left\|W_{t t}^{\prime \prime}\right\|$, we have a simple geometrical interpretation of this result. Let us define the coordinates: $X=|q|+\sum_{i=1}^{m}\left|A_{i}\right| \tau_{i}^{*}, Y=\sum_{i=1}^{m}\left\{\left|\Delta a_{i}\right|^{*}+\left|B_{i}\right| \theta_{i}^{*}\right\}, Z=\sum_{i=1}^{m}\left|\Delta b_{i}\right|^{*}$. Condition (3.13) is fulfilled for every internal point $(X, Y, Z)$ of the pyramid bounded by the planes

$$
(1+R) X+Q Y+P Z=1, X=0, Y=0, Z=0 .
$$


Remark 3.3. It is clear from inequality (3.13) that in the case, when the coefficients $a_{i}(t)$ and $b_{i}(t)(i=$ $1, \ldots, m)$ are close to constants, the second and fourth terms are small, and in the case of small delays $\theta_{i}(t)$ and $\tau_{i}(t)(i=1, \ldots, m)$, the first and third terms are small. We can make a conclusion that equation $(2.2)$ in this case preserves the property of the exponential stability of equation (3.1).

Let us formulate several tests of the exponential stability based on estimates of $\|W\|$, $\left\|W_{t}^{\prime}\right\|$ and $\left\|W_{t t}^{\prime \prime}\right\|$.

Proposition 3.4. Assume that $A^{2}>4 B$ and:

$$
\begin{aligned}
Q_{1} \equiv & \left(|q|+\sum_{i=1}^{m}\left|A_{i}\right| \tau_{i}^{*}\right)\left[\frac{2}{A+\sqrt{A^{2}-4 B}} \cdot\left\{\frac{A-\sqrt{A^{2}-4 B}}{A+\sqrt{A^{2}-4 B}}\right\}^{\frac{A-\sqrt{A^{2}-4 B}}{\sqrt{A^{2}-4 B}}}+1\right] \\
& +\sum_{i=1}^{m}\left\{\left|\Delta a_{i}\right|^{*}+\left|B_{i}\right| \theta_{i}^{*}\right\}\left[\frac{4}{A+\sqrt{A^{2}-4 B}} \cdot\left\{\frac{A-\sqrt{A^{2}-4 B}}{A+\sqrt{A^{2}-4 B}}\right\}^{\frac{A-\sqrt{A^{2}-4 B}}{2 \sqrt{A^{2}-4 B}}}\right] \\
& +\sum_{i=1}^{m}\left|\Delta b_{i}\right|^{*} \frac{1}{B}<1 .
\end{aligned}
$$

Then equation (2.2) is uniformly exponentially stable, and the difference of solution $x(t)$ of homogeneous equation (2.2) and the solution $X(t)$ of non-homogeneous equation (1.1) with the same initial conditions satisfies the inequality $|x(t)-X(t)|<\frac{\|W\|}{1-Q_{1}} f^{*}$.

Proposition 3.5. Assume that $A^{2}=4 B$ and:

$$
\begin{aligned}
Q_{2} \equiv & \left(|q|+\sum_{i=1}^{m}\left|A_{i}\right| \tau_{i}^{*}\right)\left[2+\frac{A}{4}-\left(1-\frac{A}{4}\right) \frac{1}{e^{2}}\right]+\sum_{i=1}^{m}\left\{\left|\Delta a_{i}\right|^{*}+\left|B_{i}\right| \theta_{i}^{*}\right\} \frac{4}{A e} \\
& +\sum_{i=1}^{m}\left|\Delta b_{i}\right|^{*} \frac{1}{B}<1,
\end{aligned}
$$

then equation (2.2) is uniformly exponentially stable, and the difference of solution $x(t)$ of homogeneous equation (2.2) and the solution $X(t)$ of non-homogeneous equation (1.1) with the same initial conditions satisfies the inequality $|x(t)-X(t)|<\frac{\|W\|}{1-Q_{2}} f^{*}$.

Proposition 3.6. Assume that $A^{2}<4 B$ and:

$$
\begin{aligned}
Q_{3} \equiv & \left(|q|+\sum_{i=1}^{m}\left|A_{i}\right| \tau_{i}^{*}\right)\left[1+\frac{2 \exp \left[-\frac{A}{\sqrt{4 B-A^{2}}}\left(\pi+2 \arctan \frac{\sqrt{4 B-A^{2}}}{A}\right)\right]}{1-\exp \left[-\frac{A}{\sqrt{4 B-A^{2}}} \pi\right]}\right] \\
& +\sum_{i=1}^{m}\left\{\left|\Delta a_{i}\right|^{*}+\left|B_{i}\right| \theta_{i}^{*}\right\}\left[\frac{2}{\sqrt{B}} \cdot \frac{\exp \left[-\frac{A}{\sqrt{4 B-A^{2}}}\left(\pi+\arctan \frac{\sqrt{4 B-A^{2}}}{A}\right)\right]}{1-\exp \left(-\frac{A}{\sqrt{4 B-A^{2}}} \pi\right)}\right] \\
& +\sum_{i=1}^{m}\left|\Delta b_{i}\right|^{*} \frac{1}{B} \frac{1+\exp \left(-\frac{\pi A}{\sqrt{4 B-A^{2}}}\right)}{1-\exp \left(-\frac{\pi A}{\sqrt{4 B-A^{2}}}\right)}<1,
\end{aligned}
$$


then equation (2.2) is uniformly exponentially stable, and the difference of solution $x(t)$ of homogeneous equation (2.2) and the solution $X(t)$ if non-homogeneous equation (1.1) with the same initial conditions satisfies the inequality $|x(t)-X(t)|<\frac{\|W\|}{1-Q_{3}} f^{*}$.

Remark 3.7. In the paper [3], the exponential stability of neutral equations was obtained under the assumption $A^{2} \geq 4 B$. In Proposition 3.6 in contrast with this assumption, the case of $A^{2}<4 B$ is considered. This means that we do not limit our analysis only by the case of nonoscillatory equation $x^{\prime \prime}+A x^{\prime}+B x=0$ and consider also the case of oscillating model equation.

\section{DESCRIPTION OF NEURAL-MECHANICAL MODEL OF HUMAN BALANCING}

Let us explain how second order delay equation appears in the model of balancing. The neural-mechanical model of human balancing in the sagittal plane is depicted in Figure 1 which was constructed in [23]. If we consider point $A$ as fixed point we can model the human body as an inverted pendulum with mass $m$, moment of inertia $J_{A}$ with respect to pivot $A$, while $l$ stands for the distance between the center of gravity $C$ and pivot $A$. $b$ is the torsional dashpot of damping and $k$ is the torsional spring of stiffness. The torque $Q$ is regulated by the central nervous system based on the sensory signals about rotation angle $\varphi$, angular velocity $\dot{\varphi}$ and angular acceleration $\ddot{\varphi}$ of the human body. By using the Newton second law for the moment at fixed point A we will obtain the following equation

$$
J_{A} \ddot{\varphi}(t)-m g l \sin \varphi(t)=-Q(t)
$$

where

$$
Q(t)=Q_{p}(t)+Q_{a}(t)+f(t)
$$

$Q_{p}$ is a passive torque, $Q_{a}$ - an active torque, $f(t)$ defines corresponding torques which is implied by actions which seem negligible and is not usually taken into account in the construction of the model (for example, a person standing on a slowly moving belt or overboard, a weak wind that increases resistance to motion, etc).

The passive torque is defined as

$$
Q_{p}(t)=b(t) \dot{\varphi}\left(t-\theta_{1}(t)\right)+k(t) \varphi\left(t-\theta_{2}(t)\right) .
$$

In physiological systems, the coefficients $b(t), k(t)$ are rather periodic than constant and small delays $\theta_{1}(t), \theta_{2}(t)$ exist. In [23] $\theta_{1}$ and $\theta_{2}$ are considered as negligible and the coefficients $b(t)=b, k(t)=k$ are constants.

The active torque $Q_{a}(t)$ is assumed in the nonlinear form

$$
Q_{a}(t)=\alpha \tanh \left(\frac{1}{\alpha} Q_{c}(t)\right)
$$

where $\alpha$ denotes the limit of the active torque and its linear part is

$$
Q_{c}(t)=K_{p} \varphi\left(t-\tau_{1}(t)\right)+K_{d} \dot{\varphi}\left(t-\tau_{2}(t)\right)+K_{a} \ddot{\varphi}\left(t-\tau_{3}(t)\right)
$$

with $K_{p}, K_{d}$ and $K_{a}$ being the positive constants describing controls. In physiological systems they are rather almost periodic functions than constants. $\tau_{1}, \tau_{2}, \tau_{3}$ are delays of these torques respectively. The delayed signals of angle $\varphi$ and angular velocity $\dot{\varphi}$ are provided by the vestibular system and proprioceptors, while the angular acceleration $\ddot{\varphi}$ signal is related to the information coming from the mechanoreceptors according to Newton's Second Law [14]. The saturation effect is shown in Figure 2 which is taken from [23], where the active control 


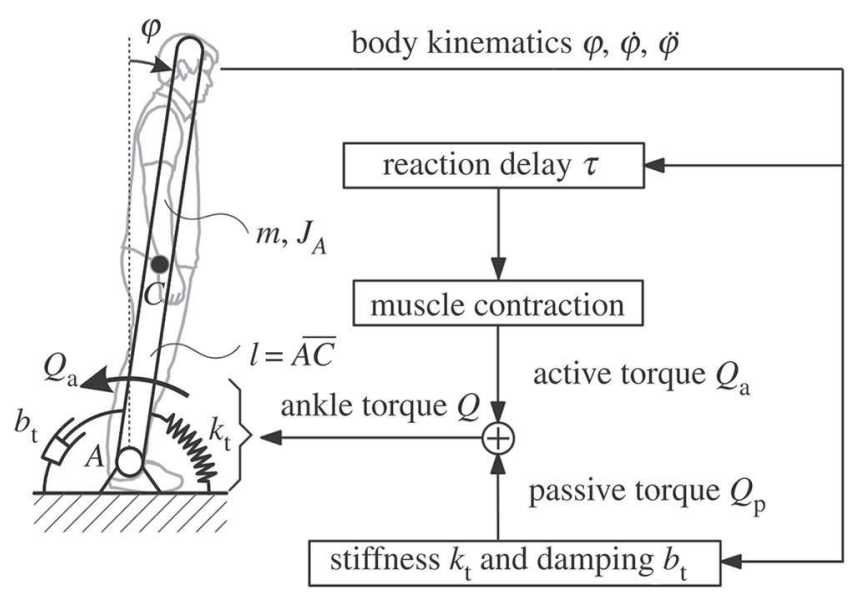

FiguRE 1. Neural-mechanical model of balancing in sagittal plane [23]

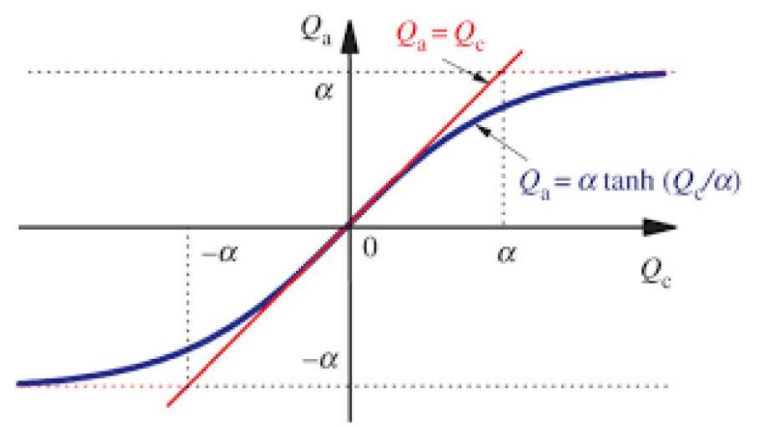

FiguRE 2. Saturated active control torque $Q_{a}$ versus linear active control torque $Q_{c}$ [23]

torque $Q_{a}$ tends to saturation torque limit $\alpha$ as $Q_{c}$ increases. The dynamics of the quiet standing process is governed by the following second-order nonlinear delay differential equation:

$$
\begin{aligned}
J_{A} \ddot{\varphi}(t) & +b(t) \dot{\varphi}\left(t-\theta_{1}(t)\right)+k(t) \varphi\left(t-\theta_{2}(t)\right)-m g l \sin \varphi(t)=-\alpha \tanh \left(\frac { 1 } { \alpha } \left(K_{p} \varphi\left(t-\tau_{1}(t)\right)\right.\right. \\
& \left.+K_{d} \dot{\varphi}\left(t-\tau_{2}(t)\right)+K_{a} \ddot{\varphi}\left(t-\tau_{3}(t)\right)\right)+f(t) .
\end{aligned}
$$

This delay differential equation is of neutral type because time delay also appears in the argument of the highest (i.e. of the second) derivative of the body angle. Let us discuss several possible directions of linearization of equation (4.6) for analysis of stability and estimates of solutions.

The trivial solution $\varphi \equiv 0$ of the nonlinear equation (4.6) corresponds to the desired equilibrium of quiet standing. Local stability can be studied on the basis of the linearized equation

$$
\begin{aligned}
& J_{A} \ddot{\varphi}(t)+b(t) \dot{\varphi}\left(t-\theta_{1}(t)\right)+k(t) \varphi\left(t-\theta_{2}(t)\right)-m g l i(t, \varphi) \varphi(t) \\
& \quad=-K_{p}(t, \varphi) \varphi\left(t-\tau_{1}(t)\right)-K_{d}(t, \varphi) \dot{\varphi}\left(t-\tau_{2}(t)\right)-K_{a}(t, \varphi) \ddot{\varphi}\left(t-\tau_{3}(t)\right)+f(t),
\end{aligned}
$$


These nonlinearities can be presented by variable coefficients in the model $i(t, \varphi)=1+\Delta i(t, y), K_{p}(t, \varphi)=$ $K_{p}+\Delta K_{p}(t, y), K_{d}(t, \varphi)=K_{d}+\Delta K_{d}(t, y), K_{a}(t, \varphi)=K_{a}+\Delta K_{a}(t, y)$, where $\Delta i(t, y) \rightarrow 0, \Delta K_{p}(t, y) \rightarrow 0$, $\Delta K_{d}(t, y) \rightarrow 0, \Delta K_{a}(t, y) \rightarrow 0$ when $y \rightarrow 0$. Note that

$$
\frac{\tanh y}{y}=1-\frac{y^{2}}{3}+\frac{y^{4}}{5}+O\left(y^{6}\right), \quad \frac{\sin \varphi}{\varphi}=1-\frac{1}{6} \varphi^{2}+\frac{1}{120} \varphi^{4}+O\left(\varphi^{6}\right) .
$$

Making the classical linearization we obtain

$$
\begin{aligned}
& J_{A} \ddot{\varphi}(t)+b \dot{\varphi}\left(t-\theta_{1}(t)\right)+k \varphi\left(t-\theta_{2}(t)\right)-m g l \varphi(t) \\
& \quad=-K_{p} \varphi\left(t-\tau_{1}(t)\right)-K_{d} \dot{\varphi}\left(t-\tau_{2}(t)\right)-K_{a} \ddot{\varphi}\left(t-\tau_{3}(t)\right)+f(t) .
\end{aligned}
$$

Formulas (4.8) allow us to obtain estimates of $\Delta i(t, \varphi), \Delta K_{p}(t, \varphi), \Delta K_{d}(t, \varphi)$ and $\Delta K_{a}(t, \varphi)$, if we have a priori estimates of the solution. Analysis of stability can be reduced to one of the homogeneous equation

$$
\begin{aligned}
\ddot{\varphi}(t) & +\frac{K_{a}}{J_{A}} \ddot{\varphi}\left(t-\tau_{3}(t)\right)+\frac{b}{J_{A}} \dot{\varphi}\left(t-\theta_{1}(t)\right)+\frac{K_{d}}{J_{A}} \dot{\varphi}\left(t-\tau_{2}(t)\right) \\
& +\frac{k}{J_{A}} \varphi\left(t-\theta_{2}(t)\right)-\frac{m g l}{J_{A}} \varphi(t)+\frac{K_{p}}{J_{A}} \varphi\left(t-\tau_{1}(t)\right)=0 .
\end{aligned}
$$

If we assume that there are no delays in reactions, then in the case of $K_{p}+k>m g l$, the second order ordinary differential equation is exponentially stable. In practice, it is known that the mechanical stiffness is insufficient for stability of human balancing [17]. The source of instability is the delay in physiological reactions. To estimate a smallness of the delays which does not break stability is the main goal in this model. We make this estimation in Section 5.

Let us take a closer look at the standard transition from equation (4.6) to linearized equations (4.7) and (4.10). Suppose the function $x^{*}(t)$ is a solution to the nonlinear equation (4.6). Is it physically achievable? It is clear that for this it must be at least Lyapunov stable. Does the exponential stability of equations (4.10), which was obtained according to the standard linearization scheme, imply exponential stability, or at least Lyapunov stability of this solution $x^{*}(t)$ ? Generally speaking, no.

Example 4.1. Let us consider the model described by nonlinear equation (4.6) with constant little strength $f$. From physical point of view, this function can be, for example, small wind. We want to be in a situation that a constant $\varphi^{*}$ is the solution. It can be achieved if all coefficients in equation (4.7) are constants. Assume that all coefficients are constant and rewrite equation (4.7) taking into account two first terms in every one of presentations (4.9) and not one term only as it was done in the classical linearization (4.10). We obtain

$$
\begin{aligned}
& J_{A} \varphi^{\prime \prime}(t)+K_{a} \varphi^{\prime \prime}\left(t-\tau_{3}(t)\right)+b \varphi^{\prime}\left(t-\theta_{1}(t)\right)+k \varphi\left(t-\theta_{1}(t)\right)-m g l \varphi(t) \\
& \quad+K_{p} \varphi\left(t-\tau_{1}(t)\right)+K_{d} \varphi^{\prime}\left(t-\tau_{2}(t)\right)-\frac{1}{\alpha^{2}} K_{p}^{3} \varphi^{3}\left(t-\tau_{1}(t)\right)+\frac{m g l}{6} \varphi^{3}(t)=f(t) .
\end{aligned}
$$

Consider equation (4.11) with the coefficients

$$
J_{A}=60, m g l=600, K_{a}=0, K_{d}=480, K_{p}=600, k=60, \alpha=0.5 .
$$

Let us set $f(t)=-60\left(\varphi^{*}\right)^{3}$, where $\varphi^{*}$ will be defined below. Assume for simplicity that

$$
\theta_{1}(t) \equiv 0, \theta_{2}(t) \equiv 0, \tau_{1}(t) \equiv 0, \tau_{2}(t) \equiv 0 \text { for } t \geq 0 .
$$


After substitution of the coefficients we obtain

$$
\varphi^{\prime \prime}(t)+8 \varphi^{\prime}(t)+\left\{\frac{K_{p}-\frac{1}{3 \alpha^{2}} K_{p}^{3}\left(\varphi^{*}\right)^{2}}{60}+\frac{10}{6}\left(\varphi^{*}\right)^{2}+1\right\} \varphi(t)=-\left(\varphi^{*}\right)^{3}
$$

where coefficients are defined by (4.12). The constant $\varphi^{*}=\frac{\sqrt{38}}{\sqrt{14399997}}$ is a solution of this equation. The coefficient of $\varphi(t)$ is negative. In this case one of the roots of the characterictic polynomial for the homogeneous equation

$$
\varphi^{\prime \prime}(t)+8 \varphi^{\prime}(t)+\left\{\frac{K_{p}-\frac{1}{3 \alpha^{2}} K_{p}^{3}\left(\varphi^{*}\right)^{2}}{60}+\frac{10}{6}\left(\varphi^{*}\right)^{2}+1\right\} \varphi(t)=0
$$

is positive and there are no even the Lyapunov stability of the solution $\varphi^{*}=\frac{\sqrt{38}}{\sqrt{14399997}}$. Instead of assumptions (4.13) we can suggest that the delays are small enough.

Denote

$$
q=\frac{K_{a}}{J_{A}}, a_{1}=\frac{b}{J_{A}}, a_{2}=\frac{K_{d}}{J_{A}}, c_{1}=\frac{k}{J_{A}}, c_{2}=\frac{-m g l}{J_{A}}, b_{1}=c_{1}+c_{2}, b_{2}=\frac{K_{p}}{J_{A}} .
$$

So we get the following equation

$$
\begin{aligned}
x^{\prime \prime}(t) & +q x^{\prime \prime}\left(t-\tau_{3}(t)\right)+a_{1} x^{\prime}\left(t-\theta_{1}(t)\right)+a_{2} x^{\prime}\left(t-\tau_{2}(t)\right)+c_{1} x\left(t-\theta_{2}(t)\right)+c_{2} x(t) \\
& +b_{2} x\left(t-\tau_{1}(t)\right)=f(t) .
\end{aligned}
$$

In the case of $\theta_{1}=0$ and $\theta_{2}=0$ we obtain

$$
x^{\prime \prime}(t)+q x^{\prime \prime}\left(t-\tau_{3}(t)\right)+a_{1} x^{\prime}(t)+a_{2} x^{\prime}\left(t-\tau_{2}(t)\right)+b_{1} x(t)+b_{2} x\left(t-\tau_{1}(t)\right)=f(t) .
$$

This equation is a particular case of equation (1.1). Thus we come after the classical linearization to the following homogeneous equation for stability studies

$$
x^{\prime \prime}(t)+q x^{\prime \prime}\left(t-\tau_{3}(t)\right)+a_{1} x^{\prime}(t)+a_{2} x^{\prime}\left(t-\tau_{2}(t)\right)+b_{1} x(t)+b_{2} x\left(t-\tau_{1}(t)\right)=0 .
$$

\section{Application to model of human BAlAnCing: estimates of Delays}

Let us study the stability of (4.17) and (4.18) on the basis of Theorem 3.1 and Propositions 3.4-3.6. The trivial solution $\varphi=0$ of (4.19) corresponds to the equilibrium of balancing. Let us describe the numerical values of several physical parameters [23]: human weight $m g=600$, length from pivot point A to center of mass C $l=1$, moment of inertia $J_{A}=60$ and passive stiffness $k=471$. The contribution of the passive dumping is usually small, it is assumed to be negligible in further analysis: $b \approx 0$. In our terms we get that $a_{1}=0$ and $b_{1}=-2.15$. We will give range for the proportional, derivative and acceleration gains $K_{p}, K_{d}$ and $K_{a}$ as follows [23]: $K_{p} \in[0,3000], K_{d} \in[0,600]$ and $K_{a} \in[-12,48]$. In our terms we get that (refer to proof of Thm 3.1 for more details) $a_{2} \in[0,10], A \in[0,10], b_{2} \in[0,50], B \in[0,47.85]$ and $q \in[-0.2,0.8]$. To study the balancing of inactive people, we will choose the maximum values for the demonstration. We will also choose the reaction delays to be fixed at $\tau_{1} \in[0,1]$ and $\tau_{2} \in[0,1]$.

Below we use $A$ and $B$ defined by (3.12) in the case $m=2$

$$
A=a_{1}+a_{2}, \quad B=b_{1}+b_{2},
$$




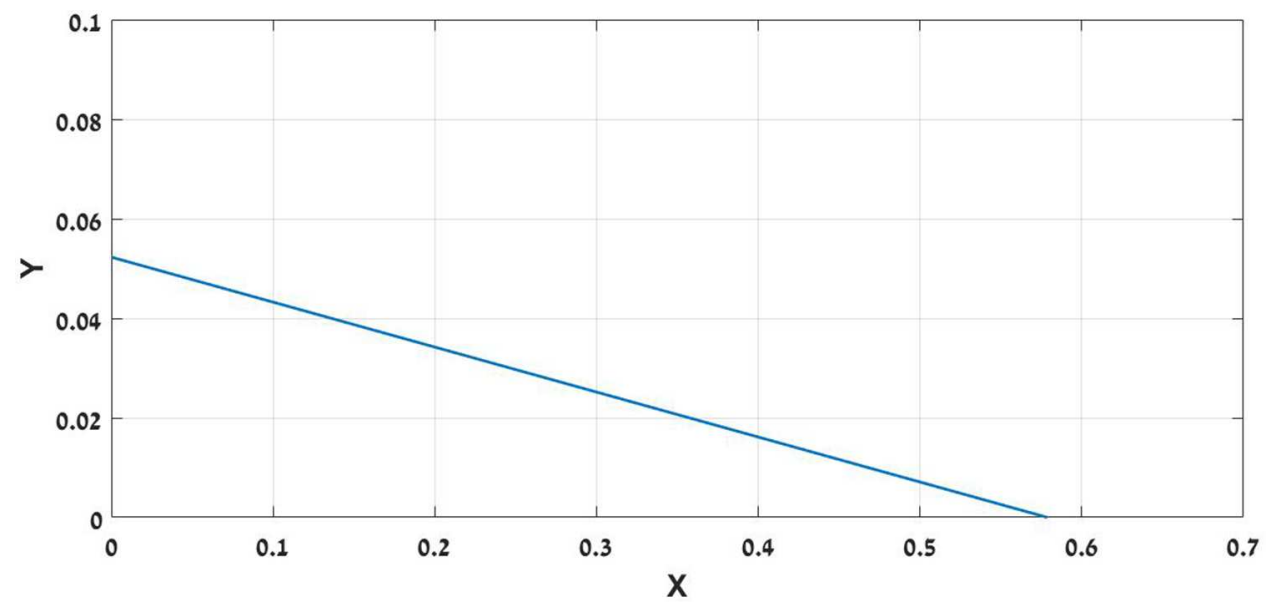

Figure 3. Domain of stability is below the blue line: $1.728 X+19.125 Y<1$.

According to Theorem 3.1, the inequality that implies exponential stability for equation (4.19) is

$$
a_{2} \tau_{2}^{*}\left[|| W_{t t}^{\prime \prime}||+1\right]+b_{2} \tau_{1}^{*}|| W_{t}^{\prime}||+|q|\left[|| W_{t t}^{\prime \prime}||+1\right]<1 .
$$

By denoting $X=\tau_{1}^{*}, Y=\tau_{2}^{*}$, we obtain

$$
b_{2}\left\|W_{t}^{\prime}\right\| X+a_{2}\left[\left\|W_{t t}^{\prime \prime}\right\|+1\right] Y<1-|q|\left[\left\|W_{t t}^{\prime \prime}\right\|+1\right] .
$$

Substituting all the terms described in the end of the previous section, namely $m g l=600, J_{A}=60, k=471$, $b=0, K_{p}=3000, K_{d}=600$ and $K_{a}=48$, we obtain the case of $A^{2}<4 B$. We will evaluate $\left\|W_{t}^{\prime}\right\|$ and $\left\|W_{t t}^{\prime \prime}\right\|$ by Proposition 3.6. We can get the area of stability below the blue line at Figure 3 .

However, we can also consider the cases where $\theta_{1}$ and $\theta_{2}$ are not zeros (this case can be important, for example, for people with disabilities). If we use the same steps of the proof like in Theorem 3.1 (see Sect. 7) we get that the following inequality

$$
a_{1} \theta_{1}^{*}\left[\left\|W_{t t}^{\prime \prime}\right\|+1\right]+a_{2} \tau_{2}^{*}\left[\left\|W_{t t}^{\prime \prime}\right\|+1\right]+c_{1} \theta_{2}^{*}\left\|W_{t}^{\prime}\right\|+b_{2} \tau_{1}^{*}\left\|W_{t}^{\prime}\right\|+|q|\left[\left\|W_{t t}^{\prime \prime}\right\|+1\right]<1
$$

implies the exponential stability for equation (4.17). Note that from the mechanical point of view $a_{1}$ is sufficiently small.

By denoting $X=\tau_{1}^{*}, Y=\tau_{2}^{*}, Z=\theta_{2}^{*}$ we obtain

$$
b_{2}\left\|W_{t}^{\prime}\right\| X+a_{2}\left[\left\|W_{t t}^{\prime \prime}\right\|+1\right] Y+c_{1}\left\|W_{t}^{\prime}\right\| Z<1-\left\{|q|+a_{1} \theta_{1}^{*}\right\}\left[\left\|W_{t t}^{\prime \prime}\right\|+1\right]
$$

We can see that for small $\theta_{2}$ we get similar results to inequality (5.3). If $\theta_{2}$ is variable, for example $0 \leq \theta_{2}(t) \leq 1$, we can see at Figure 4 that the area of stability is inside the pyramid.

\section{EXAmples on estimates of ACCELERATION COEFFiCient $q$}

Let us demonstrate few examples that allows us to estimate $q$ in the human balancing model.

Example 6.1. The inequality

$$
A^{2}>4 B
$$




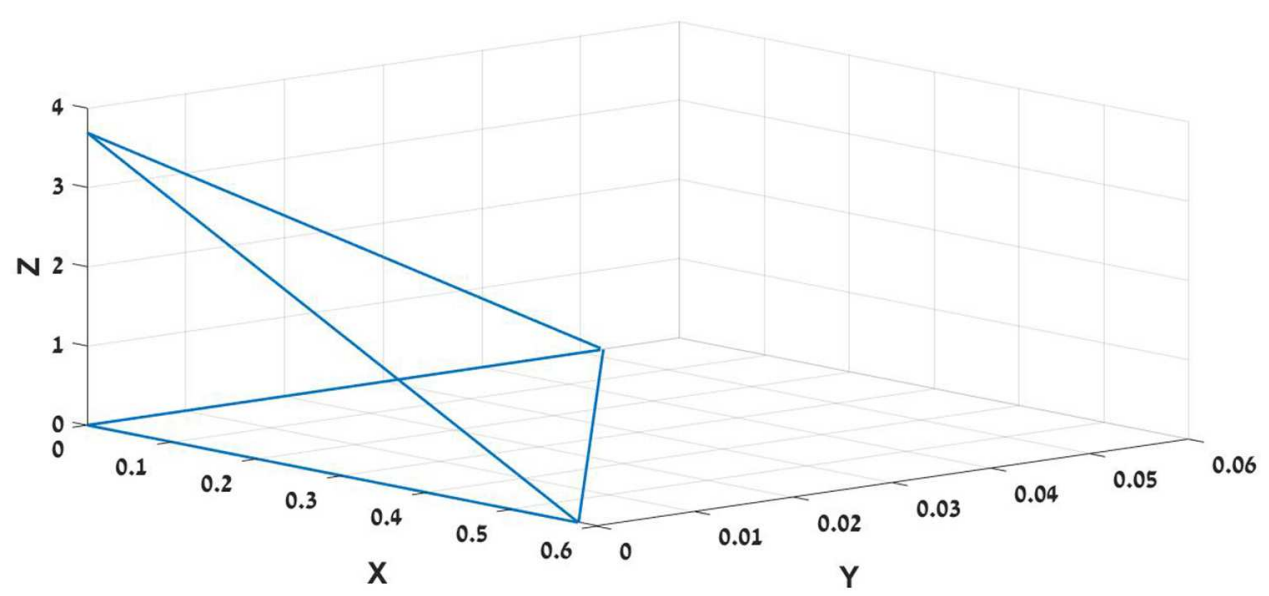

Figure 4 . Domain of stability with $\theta_{2}$ is inside the pyramid: $1.728 X+19.125 Y+0.271 Z<1$.

means that

$$
\left(\frac{b+K_{d}}{J_{a}}\right)^{2}>4\left(\frac{k-m g l+K_{p}}{J_{a}}\right)
$$

In this case we will need to use Proposition 3.4 for the stability analysis. Let us take numbers that match this case: $A=8$ and $B=7.85$. Now if we substitute the physical parameters (see Sect. 4 for details) in Proposition 3.4 we will get the following inequality

$$
\begin{aligned}
& \left(\frac{K_{d}}{J_{a}} \tau_{1}^{*}+|q|\right)\left[1+\frac{2}{\frac{b+K_{d}}{J_{a}}+\sqrt{\left(\frac{b+K_{d}}{J_{a}}\right)^{2}-4 \frac{k-m g l+K_{p}}{J_{a}}}}\right. \\
& \left.\times\left(\frac{\frac{b+K_{d}}{J_{a}}-\sqrt{\left(\frac{b+K_{d}}{J_{a}}\right)^{2}-4 \frac{k-m g l+K_{p}}{J_{a}}}}{\frac{b+K_{d}}{J_{a}}+\sqrt{\left(\frac{b+K_{d}}{J_{a}}\right)^{2}-4 \frac{k-m g l+K_{p}}{J_{a}}}}\right) \frac{\frac{b+K_{d}}{J_{a}}-\sqrt{\left(\frac{b+K_{d}}{J_{a}}\right)^{2}-4 \frac{k-m g l+K_{p}}{J_{a}}}}{\sqrt{\left(\frac{b+K_{d}}{J_{a}}\right)^{2}-4 \frac{k-m g l+K_{p}}{J_{a}}}}\right] \\
& +\left(\frac{K_{p}}{J_{a}} \tau_{2}^{*}\right)\left[\frac{4}{\frac{b+K_{d}}{J_{a}}+\sqrt{\left(\frac{b+K_{d}}{J_{a}}\right)^{2}-4 \frac{k-m g l+K_{p}}{J_{a}}}} .\right. \\
& \left.\times\left(\frac{\frac{b+K_{d}}{J_{a}}-\sqrt{\left(\frac{b+K_{d}}{J_{a}}\right)^{2}-4 \frac{k-m g l+K_{p}}{J_{a}}}}{\frac{b+K_{d}}{J_{a}}+\sqrt{\left(\frac{b+K_{d}}{J_{a}}\right)^{2}-4 \frac{k-m g l+K_{p}}{J_{a}}}}\right)^{\frac{\frac{b+K_{d}}{J_{a}}-\sqrt{\left(\frac{b+K_{d}}{J_{a}}\right)^{2}-4 \frac{k-m g l+K_{p}}{J_{a}}}}{2 \sqrt{\left(\frac{b+K_{d}}{J_{a}}\right)^{2}-4 \frac{k-m g l+K_{p}}{J_{a}}}}}\right]<1 .
\end{aligned}
$$


Now by substituting $m g l=600, J_{a}=60, k=471, b=0, K_{d}=480, K_{p}=600$ and $\tau_{1}^{*}=\tau_{2}^{*}=0.05$ (see Sect. 4 for details) we can evaluate the range of $q$ :

$$
|q|<0.1
$$

Example 6.2. The inequality

$$
A^{2}=4 B
$$

means that

$$
\left(\frac{b+K_{d}}{J_{a}}\right)^{2}=4\left(\frac{k-m g l+K_{p}}{J_{a}}\right)
$$

In this case we will need to use Proposition 3.5 for the stability analysis. Let us take numbers that match this case: $A=4$ and $B=4$. Now if we substitute the physical parameters (see Sect. 4 for details) in Proposition 3.5 we will get the following inequality

$$
\left(\frac{K_{d}}{J_{a}} \tau_{1}^{*}+|q|\right)\left[2+\frac{b+K_{d}}{4 J_{a}}-\left(1-\frac{b+K_{d}}{4 J_{a}}\right) \frac{1}{e^{2}}\right]+K_{p} \tau_{2}^{*} \frac{4}{e\left(b+K_{d}\right)}<1 .
$$

Now by substituting $m g l=600, J_{a}=60, k=471, b=0, K_{d}=240, K_{p}=369$ and $\tau_{1}^{*}=\tau_{2}^{*}=0.05$ (see Sect. 4 for details) we can evaluate the range of $q$ :

$$
|q|<0.1343
$$

Example 6.3. The inequality

$$
A^{2}<4 B
$$

means that

$$
\left(\frac{b+K_{d}}{J_{a}}\right)^{2}<4\left(\frac{k-m g l+K_{p}}{J_{a}}\right)
$$

In this case we will need to use Proposition 3.6 for the stability analysis. Let us take numbers that match this case: $A=10$ and $B=40$. Now if we substitute the physical parameters (see Sect. 4 for details) in Proposition 3.6 we will get the following inequality

$$
\left(\frac{K_{d}}{J_{a}} \tau_{1}^{*}+|q|\right)\left[1+\frac{2 \exp \left[-\frac{\frac{b+K_{d}}{J_{a}}}{\sqrt{4 \frac{k-m g l+K_{p}}{J_{a}}-\left(\frac{b+K_{d}}{J_{a}}\right)^{2}}}\left(\pi+2 \arctan \frac{\left.\left.\sqrt{4 \frac{k-m g l+K_{p}}{J_{a}}-\left(\frac{b+K_{d}}{J_{a}}\right)^{2}}\right)\right]}{\frac{b+K_{d}}{J_{a}}}\right)\right]}{1-\exp \left[-\frac{\pi \frac{b+K_{d}}{J_{a}}}{\sqrt{4 \frac{k-m g l+K_{p}}{J_{a}}-\left(\frac{b+K_{d}}{J_{a}}\right)^{2}}}\right]}\right]
$$




$$
+\frac{K_{p}}{J_{a}} \tau_{2}^{*}\left[\frac{2 \sqrt{J_{a}}}{\sqrt{k-m g l+K_{p}}} \frac{\exp \left[-\frac{\frac{b+K_{d}}{J_{a}}}{\sqrt{4 \frac{k-m g l+K_{p}}{J_{a}}-\left(\frac{b+K_{d}}{J_{a}}\right)^{2}}}\left(\pi+\arctan \frac{\left.\sqrt{4 \frac{k-m g l+K_{p}}{J_{a}}-\left(\frac{b+K_{d}}{J_{a}}\right)^{2}}\right)}{\frac{b+K_{d}}{J_{a}}}\right)\right]}{1-\exp \left[-\frac{\pi \frac{b+K_{d}}{J_{a}}}{\sqrt{4 \frac{k-m g l+K_{p}}{J_{a}}-\left(\frac{b+K_{d}}{J_{a}}\right)^{2}}}\right]}\right]<1 .
$$

Now by substituting $m g l=600, J_{a}=60, k=471, b=0, K_{d}=600, K_{p}=2529$ and $\tau_{1}^{*}=\tau_{2}^{*}=0.05$ (see Sect. 4 for details) we can evaluate the range of $q$ :

$$
|q|<0.4886
$$

\section{ProOfS}

In the begining of Section 3 we defined the Cauchy function $W(t, s)$ of ordinary differential equation (3.1). This actually describes how this function $W(t, s)$ for every fixed $s$ can be constructed as the solutions of equation (3.2) satisfying (3.3). Constructing $W(t, s)$ in every one of the noted cases $A^{2}>4 B, A^{2}=4 B$ and $A^{2}<4 B$

and then integrating with respect to $s$, we get the following three assertions allowing us to obtain $\|W\|,\left\|W_{t}^{\prime}\right\|$ and $\left\|W_{t t}^{\prime \prime}\right\|$ defined by formulas (3.10) and (3.11).

Lemma 7.1. Let the coefficients $A$ and $B$ defined by (3.12) satisfy the inequalities $A>0, B>0$ and $A^{2}>4 B$. Then

$$
\begin{gathered}
\|W\|=\frac{1}{B}, \\
\left\|W_{t}^{\prime}\right\|=\frac{4}{A+\sqrt{A^{2}-4 B}} \cdot\left\{\frac{A-\sqrt{A^{2}-4 B}}{A+\sqrt{A^{2}-4 B}}\right\}^{\frac{A-\sqrt{A^{2}-4 B}}{2 \sqrt{A^{2}-4 B}}}, \\
\left\|W_{t t}^{\prime}\right\|=1+\frac{2}{A+\sqrt{A^{2}-4 B}} \cdot\left\{\frac{A-\sqrt{A^{2}-4 B}}{A+\sqrt{A^{2}-4 B}}\right\}^{\frac{A-\sqrt{A^{2}-4 B}}{\sqrt{A^{2}-4 B}}} .
\end{gathered}
$$

Lemma 7.2. Let the coefficients $A$ and $B$ defined by (3.12) satisfy the inequalities $A>0, B>0$ and $A^{2}=4 B$. Then

$$
\begin{gathered}
\|W\|=\frac{1}{B}, \\
\left\|W_{t}^{\prime}\right\|=\frac{4}{A e}, \\
\left\|W_{t t}^{\prime}\right\|=1+\frac{A}{4}-\left(1-\frac{A}{4}\right) \frac{1}{e^{2}} .
\end{gathered}
$$


Lemma 7.3. Let the coefficients $A$ and $B$ defined by (3.12) satisfy the inequalities $A>0, B>0$ and $A^{2}<4 B$. Then

$$
\begin{gathered}
\|W\|=\frac{1}{B} \cdot \frac{1+\exp \left[-\frac{A}{\sqrt{4 B-A^{2}}} \pi\right]}{1-\exp \left[-\frac{A}{\sqrt{4 B-A^{2}}} \pi\right]}, \\
\left\|W_{t}^{\prime}\right\|=\frac{2}{\sqrt{B}} \cdot \frac{\exp \left[-\frac{A}{\sqrt{4 B-A^{2}}}\left(\pi+\arctan \frac{\sqrt{4 B-A^{2}}}{A}\right)\right]}{1-\exp \left[-\frac{A}{\sqrt{4 B-A^{2}}} \pi\right]}, \\
\left\|W_{t t}^{\prime}\right\|=\frac{2 \exp \left[-\frac{A}{\sqrt{4 B-A^{2}}}\left(\pi+2 \arctan \frac{\sqrt{4 B-A^{2}}}{A}\right)\right]}{1-\exp \left[-\frac{A}{\sqrt{4 B-A^{2}}} \pi\right]} .
\end{gathered}
$$

Proof of Theorem 3.1. Consider the following equation

$$
x^{\prime \prime}(t)+q(t) x^{\prime \prime}\left(r_{i}(t)\right)+\sum_{i=1}^{m} a_{i}(t) x^{\prime}\left(g_{i}(t)\right)+\sum_{i=1}^{m} b_{i}(t) x\left(h_{i}(t)\right)=f(t), \quad t \in[0, \infty),
$$

where $r_{i}(t)=t-\phi_{i}(t), g_{i}(t)=t-\tau_{i}(t)$ and $h_{i}(t)=t-\theta_{i}(t)$ with the zero initial functions

$$
x(\xi)=x^{\prime}(\xi)=x^{\prime \prime}(\xi)=0 \text { for } \xi<0 .
$$

It is known [1] that in the analysis of stability, we can consider only the zero initial values

$$
x(0)=x^{\prime}(0)=x^{\prime \prime}(0)=0 .
$$

Let us start with the case of $g_{i}(t) \geq 0, h_{i}(t) \geq 0$. We can rewrite equation (7.10):

$$
\begin{aligned}
x^{\prime \prime}(t)+q(t) x^{\prime \prime}\left(r_{i}(t)\right)+A x^{\prime}(t)-A x^{\prime}(t) & +\sum_{i=1}^{m} a_{i}(t) x^{\prime}\left(g_{i}(t)\right)+B x(t)-B x(t) \\
& +\sum_{i=1}^{m} b_{i}(t) x\left(h_{i}(t)\right)=f(t)
\end{aligned}
$$

and

$$
\begin{aligned}
x^{\prime \prime}(t)+A x^{\prime}(t)+B x(t) & -\sum_{i=1}^{m} A_{i} \int_{g_{i}(t)}^{t} x^{\prime \prime}(s) \mathrm{d} s-\sum_{i=1}^{m} \Delta a_{i}(t) x^{\prime}\left(g_{i}(t)\right)-\sum_{i=1}^{m} B_{i} \int_{h_{i}(t)}^{t} x^{\prime}(s) \mathrm{d} s \\
& -\sum_{i=1}^{m} \Delta b_{i}(t) x\left(h_{i}(t)\right)+q(t) x^{\prime \prime}\left(r_{i}(t)\right)=f(t), \quad t \in[0,+\infty) .
\end{aligned}
$$


Let us make the Azbelev $W$-transform [1], substituting

$$
\begin{gathered}
x(t)=\int_{0}^{t} W(t, s) z(s) \mathrm{d} s, \\
x^{\prime}(t)=\int_{0}^{t} W_{t}^{\prime}(t, s) z(s) \mathrm{d} s, \\
x^{\prime \prime}(t)=\int_{0}^{t} W_{t t}^{\prime \prime}(t, s) z(s) \mathrm{d} s+z(t) .
\end{gathered}
$$

where $z \in L_{\infty}\left(L_{\infty}\right.$ in the space of essentially bounded functions $\left.z:[0, \infty) \rightarrow(-\infty, \infty)\right)$, in equation (7.14).

We obtain the following equation:

$$
z(t)=(K z)(t)+f(t)
$$

where the operator $K: L_{\infty} \rightarrow L_{\infty}$ is defined by the equality

$$
(K z)(t)=\left\{\begin{array}{l}
\sum_{i=1}^{m} A_{i} \int_{g_{i}(t)}^{t}\left\{\int_{0}^{s} W_{s s}^{\prime \prime}(s, \xi) z(\xi) d \xi+z(s)\right\} \mathrm{d} s \\
\sum_{i=1}^{m} \Delta a_{i}(t) \int_{0}^{g_{i}(t)} W_{t}^{\prime}\left(g_{i}(t), s\right) z(s) \mathrm{d} s \\
\sum_{i=1}^{m} B_{i} \int_{h_{i}(t)}^{t} \int_{0}^{s} W_{s}^{\prime}(s, \xi) z(\xi) d \xi \mathrm{d} s \\
\sum_{i=1}^{m} \Delta b_{i}(t) \int_{0}^{h_{i}(t)} W\left(h_{i}(t), s\right) z(s) \mathrm{d} s \\
q(t) \sigma\left(r_{i}(t)\right)\left\{\int_{0}^{t} W_{t t}^{\prime \prime}(t, s) z(s) \mathrm{d} s+z(t)\right\}
\end{array}\right.
$$

where

$$
\sigma(t)=\left\{\begin{array}{ll}
1, & t \geq 0 \\
0, & t<0
\end{array} .\right.
$$

Define the operator $|K|: L_{\infty} \rightarrow L_{\infty}$

$$
(|K| z)(t)=\left\{\begin{array}{l}
\sum_{i=1}^{m}\left|A_{i}\right| \int_{g_{i}(t)}^{t}\left\{\int_{0}^{s}\left|W_{s s}^{\prime \prime}(s, \xi)\right| z(\xi) d \xi+z(s)\right\} \mathrm{d} s \\
\sum_{i=1}^{m}\left|\Delta a_{i}(t)\right| \int_{0}^{g_{i}(t)}\left|W_{t}^{\prime}\left(g_{i}(t), s\right)\right| z(s) \mathrm{d} s \\
\sum_{i=1}^{m}\left|B_{i}\right| \int_{h_{i}(t)}^{t} \int_{0}^{s}\left|W_{s}^{\prime}(s, \xi)\right| z(\xi) d \xi \mathrm{d} s \\
\sum_{i=1}^{m}\left|\Delta b_{i}(t)\right| \int_{0}^{h_{i}(t)}\left|W\left(h_{i}(t), s\right)\right| z(s) \mathrm{d} s \\
|q(t)| \sigma\left(r_{i}(t)\right)\left\{\int_{0}^{t}\left|W_{t t}^{\prime \prime}(t, s)\right| z(s) \mathrm{d} s+z(t)\right\}
\end{array}\right.
$$

Let us denote by $\|K\|$ the norm of the operator $|K|: L_{\infty} \rightarrow L_{\infty}$.

The inequality (3.13) implies that $\|K\|<1$, so there exists the bounded operator $(I-K)^{-1}: L_{\infty} \rightarrow L_{\infty}$ such that for every bounded right hand side $f(t)$, the solution $z(t)$ is bounded on the semiaxis. Then $x(t)$ which is equal to $\int_{0}^{\infty} W(t, s) z(s) \mathrm{d} s$ is also bounded. Now according to the Bohl-Perron theorem [1] the solution of the homogeneous equation and the Cauchy function $C(t, s)$ satisfy the exponential estimate $(2.6)$. 
In the general case (i.e. without the assumption $g_{i}(t) \geq 0, h_{i}(t) \geq 0$ for $t \geq 0$ ) we can write equation (7.14) in the form

$$
\begin{aligned}
x^{\prime \prime}(t) & +A x^{\prime}(t)+B x(t)-\sum_{i=1}^{m} A_{i} \sigma\left(g_{i}(t)\right) \int_{g_{i}(t)}^{t} x^{\prime \prime}(s) \mathrm{d} s-\sum_{i=1}^{m} \Delta a_{i}(t) x^{\prime}\left(g_{i}(t)\right) \\
& -\sum_{i=1}^{m} B_{i} \sigma\left(h_{i}(t)\right) \int_{h_{i}(t)}^{t} x^{\prime}(s) \mathrm{d} s-\sum_{i=1}^{m} \Delta b_{i}(t) x\left(h_{i}(t)\right)+q(t) x^{\prime \prime}\left(r_{i}(t)\right)=\tilde{f}(t), \quad t \in[0,+\infty),
\end{aligned}
$$

where

$$
\tilde{f}(t)=f(t)+\sum_{i=1}^{m} A_{i}\left(1-\sigma\left(g_{i}(t)\right) \int_{g_{i}(t)}^{t} x^{\prime \prime}(s) \mathrm{d} s+\sum_{i=1}^{m} B_{i}\left(1-\sigma\left(h_{i}(t)\right) \int_{h_{i}(t)}^{t} x^{\prime}(s) \mathrm{d} s .\right.\right.
$$

It is clear that $\tilde{f}(t)$ is bounded. The exponential estimates of the Cauchy function in the first case imply the boundedness of solution $x(t)$ for $t \in[0, \infty)$ for every bounded on the semiaxis $[0, \infty)$ right-hand side $f(t)$. The Bohl-Perron theorem implies the exponential estimate of the Cauchy function and nontrivial solutions. It follows from the fact $\|K\|<1$ that $|z| \leq \frac{1}{1-Q}$ and $|x(t)| \leq \int_{0}^{t}|W(t, s)| z(s) \mathrm{d} s \leq \frac{\|W\|}{1-Q} f^{*}$. This completes the proof.

Proofs of Propositions 3.4-3.6 follow now from Theorem 3.1 and Lemmas 7.1-7.3, respectively.

\section{OpEn questions}

It would be interesting to develop our approach to the study of stability of the neutral delay differential equation of the order $n$ :

$$
x^{(n)}+\sum_{i=1}^{m} q_{i}(t) x^{(n)}\left(t-\theta_{n}^{i}(t)\right)+\sum_{i=1}^{m} a_{n-1}^{i} x^{(n-1)}\left(t-\theta_{n-1}^{i}(t)\right)+\ldots+\sum_{i=1}^{m} a_{0}^{i} x\left(t-\theta_{0}^{i}(t)\right)=0
$$

Systems of second order equations could be studied on the basis of the construction of the Cauchy matrix. Such systems describe model in which a motion of point objects are studied. In practice, usually 3-dimensional systems have to be considered.

It would be interesting to consider the neutral equation

$$
x^{\prime \prime}(t)-\sum_{i=1}^{m} q_{i}(t) x^{\prime \prime}\left(t-\phi_{i}(t)\right)+\sum_{i=1}^{m} b_{i}(t) x\left(t-\theta_{i}(t)\right)=f(t),
$$

without damping terms (i.e. without the first derivative $x^{\prime}\left(t-\tau_{i}(t)\right)$ ). Asymptotic properties of equation (8.2) without damping term (i.e. in the case of $a_{i}(t) \equiv 0$ for $t \in[0, \infty)$ ) were studied in ([19], Chapter III, Section 16 , pp. 105-106), were instability of the equation $x^{\prime \prime}(t)+b x(t-\theta)=0$, for every pair of positive constants $b$ and $\theta$ was obtained. Conditions of stability of second order equations with variable coefficients and delays were obtained in [7].

Results about boundedness of solutions for vanishing delays $\left(\theta_{i}(t) \rightarrow 0\right.$ for $\left.t \rightarrow \infty\right)$ and about asymptotic representations of solutions were obtained in [15, 20], see also ([19], Chapter III, Section 16). Boundedness of solutions for equations with advanced arguments $\left(\theta_{i}(t) \leq 0\right)$ was studied in [8]. Results on the exponential stability of the equation $x^{\prime \prime}(t)+a x(t)-b x(t-\theta)=0$ with constant coefficients and delay were obtained in [5]. First results based on nonoscillation for the exponential stability of the second order equation without damping term and with variable coefficients and delays were obtained in the paper [6]. 
Acknowledgements. The second author Shai Levi would like to thank Department of Advanced Studies of Ariel University for the scholarship and help. Also, this paper is part of Shai Levi PhD thesis.

\section{FUNDING}

Non-relevant.

\section{REFERENCES}

[1] N.V. Azbelev, V.P. Maksimov and L.F. Rakhmatullina, Introduction to theory of functional-differential equations. Nauka, Moscow (1991) (in Russian).

[2] N.V. Azbelev and P.M. Simonov, Stability of differential equations with aftereffect. Stability and Control: Theory, Methods and Applications, 20. Taylor and Francis, London (2003).

[3] L. Berezansky and A. Domoshnitsky, On stability of the second order neutral differential equation. Appl. Math. Lett. 88 (2019) 90-95.

[4] T.A. Burton, Stability by fixed point theory for functional differential equations. Dover Publications, Mineola, New York (2006).

[5] B. Cahlon and D. Schmidt, Stability criteria for second-order delay differential equations with mixed coefficients. J. Comput. Appl. Math. 170 (2004) 79-102.

[6] A. Domoshnitsky, Nonoscillation, maximum principles and exponential stability of second order delay differential equations without damping term. J. Inequal. Appl. 2014 (2014) 361.

[7] A. Domoshnitsky, Unboundedness of solutions and instability of differential equations of the second order with delayed argument. Differ. Integr. Equ. 14 (2001) 559-576.

[8] Z. Došla and I.T. Kiguradze, On boundedness and stability of solutions of second order linear differential equations with advanced arguments. Adv. Math. Sci. Appl. Gakkotosho, Tokyo 9 (1999) 1-24.

[9] L.N. Erbe, Q. Kong and B.G. Zhang, Oscillation theory for functional differential equations. Dekker, New York/Basel (1995).

[10] T. Erneux, Applied delay differential equations. Springer Science + Business Media (2009).

[11] V.N. Fomin, A.L. Fradkov and V.A. Yakubovich, Adaptive control of dynamical objects. Moscow: Nauka (1981).

[12] K. Gopalasamy, Stability and oscillation in delay differential equation of population dynamics. Kluwer Academic publishers, Dordrecht, Boston, London (1992).

[13] I. Gyori and G. Ladas, Oscillation Theory of Delay Differential Equations. Clarendon, Oxford (1991).

[14] T. Insperger, J.G. Milton and G. Stepan, Acceleration feedback improves balancing against reflex delay. J. R. Soc. Interface 10 (2013) 20120763.

[15] D.V. Izjumova, About boundedness and stability of solutions of nonlinear functional-differential equations of the second order. Proc. Georg. Acad. Sci. V. 100 (1980) 285-288 (in Russian).

[16] V. Kolmanovskii and A. Myshkis, Introduction to the theory and applications of functional differential equations. Kluwer Academic Publisher, Dordrecht/Boston/London (1999).

[17] I.D. Loram and M. Lakie, Direct measurement of human ankle stiffness during quiet standing: the intrinsic mechanical stiffness is insufficient for stability. J. Physiol. 545 (2002) 1041-1053.

[18] N. Minorski, Nonlinear oscillations. Van Nostrand, New York (1962).

[19] A.D. Myshkis, Linear differential equations with delayed argument. Moscow, Nauka, 1972, 352 p. (in Russian)

[20] M. Pinto, Asymptotic solutions for second order delay differential equations. Nonlin. Anal. : TMA 28 (1999) 1729-1740.

[21] A.V. Shatyrko and D. Ya Khusainov, Absolute interval stability of indirect regulating systems of neutral type. J. Autom. Inf. Sci. 42 (2010) 43-54.

[22] A.H. Vette, K. Masani, K. Nakazawa and M.R. Popovic, Neural-mechanical feedback control scheme generates physiological ankle torque fluctuation during quiet stance. IEEE Trans. Neural. Syst. Rehabil. Eng. 18 (2010) 86-95.

[23] L. Zhang, G. Stepan and T. Insperger, Saturation limits the contribution of acceleration feedback to balancing against reaction delay. J. R. Soc. Interface 2018 (2018) 20170771.

[24] L. Zhang and G. Stepan, Exact stability chart of an elastic beam subjected to delayed feedback. J. Sound Vib. 367 (2016) $219-232$. 